\title{
Judgment aggregation without full rationality
}

Citation for published version (APA):

Dietrich, F. K., \& List, C. (2007). Judgment aggregation without full rationality. METEOR, Maastricht University School of Business and Economics. METEOR Research Memorandum No. 023 https://doi.org/10.26481/umamet.2007023

Document status and date:

Published: 01/01/2007

DOI:

10.26481/umamet.2007023

Document Version:

Publisher's PDF, also known as Version of record

\section{Please check the document version of this publication:}

- A submitted manuscript is the version of the article upon submission and before peer-review. There can be important differences between the submitted version and the official published version of record.

People interested in the research are advised to contact the author for the final version of the publication, or visit the DOI to the publisher's website.

- The final author version and the galley proof are versions of the publication after peer review.

- The final published version features the final layout of the paper including the volume, issue and page numbers.

Link to publication

\footnotetext{
General rights rights.

- You may freely distribute the URL identifying the publication in the public portal. please follow below link for the End User Agreement:

www.umlib.nl/taverne-license

Take down policy

If you believe that this document breaches copyright please contact us at:

repository@maastrichtuniversity.nl

providing details and we will investigate your claim.
}

Copyright and moral rights for the publications made accessible in the public portal are retained by the authors and/or other copyright owners and it is a condition of accessing publications that users recognise and abide by the legal requirements associated with these

- Users may download and print one copy of any publication from the public portal for the purpose of private study or research.

- You may not further distribute the material or use it for any profit-making activity or commercial gain

If the publication is distributed under the terms of Article $25 \mathrm{fa}$ of the Dutch Copyright Act, indicated by the "Taverne" license above, 
Franz Dietrich, Christian List

Judgment aggregation without full rationality

$\mathrm{RM} / 07 / 023$

(RM/06/032 -revised-)

\section{METE@R}

Maastricht research school of Economics of TEchnology and ORganizations

Universiteit Maastricht

Faculty of Economics and Business Administration P.O. Box 616

NL - 6200 MD Maastricht

phone : ++31433883830

fax $\quad$ : ++31433884873 



\title{
Judgment aggregation without full rationality
}

\author{
Franz Dietrich and Christian List ${ }^{1}$ \\ August 2006, revised June 2007
}

\begin{abstract}
Several recent results on the aggregation of judgments over logically connected propositions show that, under certain conditions, dictatorships are the only propositionwise aggregation functions generating fully rational (i.e., complete and consistent) collective judgments. A frequently mentioned route to avoid dictatorships is to allow incomplete collective judgments. We show that this route does not lead very far: we obtain oligarchies rather than dictatorships if instead of full rationality we merely require that collective judgments be deductively closed, arguably a minimal condition of rationality, compatible even with empty judgment sets. We derive several characterizations of oligarchies and provide illustrative applications to Arrowian preference aggregation and Kasher and Rubinstein's group identification problem.
\end{abstract}

\section{Introduction}

Sparked by the "discursive paradox", the problem of judgment aggregation has recently received much attention. The paradox consists in the fact that, if a group of individuals takes majority votes on logically connected propositions, the resulting collective judgments may be inconsistent, even when all individual judgments are consistent (Pettit 2001, extending Kornhauser and Sager 1986). Condorcet's famous paradox of cyclical majority preferences is a special case of the "discursive paradox", applied to judgments of binary preferability of the form " $x$ is preferable to $y$ ", " $y$ is preferable to $z$ " and so on (List and Pettit 2004). A simple example of the general problem is given in Table 1, where $a$ and $b$ are atomic propositions and $a \wedge b$ is their conjunction.

From subsequent impossibility results we know that majority voting is not alone in its failure to ensure rational collective judgments on interconnected

\footnotetext{
${ }^{1}$ This paper was circulated in August 2006 and presented at the Yale workshop on Aggregation of Opinions, September 2006, at the Centre interuniversitaire de rechere en économie quantitative, Montreal, October 2006, and at the 1st International Workshop on Computational Social Choice, Amsterdam, December 2006. We are grateful to the participants at these occasions and the anonymous referees for comments. We also thank Ton Storcken for discussion. Elad Dokow and Ron Holzman have independently proved closely related results, which were also presented at the Yale workshop in September 2006, and circulated in the December 2006 paper (Dokow and Holzman 2006). Addresses for correspondence: C. List, Department of Government, London School of Economics, London WC2A 2AE, U.K.; F. Dietrich, Department of Quantitative Economics, University of Maastricht, P.O. Box 616, 6200 MD Maastricht, The Netherlands.
} 


\begin{tabular}{|c|c|c|c|}
\hline & $a$ & $b$ & $a \wedge b$ \\
\hline Individual 1 & True & True & True \\
\hline Individual 2 & True & False & False \\
\hline Individual 3 & False & True & False \\
\hline Majority & True & True & False \\
\hline
\end{tabular}

Table 1: A discursive paradox

propositions, where rationality is understood as the conjunction of two requirements. Consistency: the set of accepted propositions must not entail a contradiction; and completeness: it must contain a member of every propositionnegation pair under consideration. The generic finding is that dictatorships are the only propositionwise aggregation functions generating consistent and complete collective judgments and satisfying some minimal conditions (List and Pettit 2002, Pauly and van Hees 2006, Dietrich 2006, Gärdenfors 2006, Nehring and Puppe 2002, 2005, van Hees 2007, Dietrich 2007, Mongin 2005, Dokow and Holzman 2005, Dietrich and List 2007a; the precise conditions differ from result to result). This finding is broadly analogous to Arrow's theorem for preference aggregation. $^{2}$

A frequently mentioned way to avoid this impossibility is to drop the completeness requirement at the collective level and thus to allow the group to make no judgment on some propositions. Examples of aggregation functions that ensure consistency at the expense of incompleteness are unanimity and certain supermajority functions, where a proposition - and similarly its negation - is collectively accepted if and only if a particular supermajority or all of the individuals accept it (List and Pettit 2002, List 2004, Dietrich and List 2007b; the latter paper contains an analysis of anonymous judgment aggregation without full rationality).

The most forceful critique of requiring completeness has been made by Gärdenfors (2006), in line with his influential theory of belief revision (Alchourron, Gärdenfors and Makinson 1985). Describing completeness as a "strong and unnatural assumption", Gärdenfors has argued that neither individuals nor a group need to hold complete judgments and that "the [existing] impossibility results are consequences of an unnaturally strong restriction on the outcomes of a voting function". Gärdenfors has also proved the first impossibility result on judgment aggregation without completeness. His result shows that, under certain conditions, any aggregation function generating consistent and deductively closed (but not necessarily complete) collective judgments is weakly oligarchic: there is a smallest subgroup of individuals - the oligarchs - whose joint individual acceptance of any proposition is sufficient (though perhaps not necessary)

\footnotetext{
${ }^{2}$ Precursors to this recent literature are Wilson's (1975) and Rubinstein and Fishburn's (1986) contributions on abstract aggregation theory. A related literature in artificial intelligence is also concerned with merging sets of propositions, but without aiming at propositionwise aggregation (e.g., Konieczny and Pino-Perez 2002).
} 
for its collective acceptance. Deductive closure is the requirement that any proposition under consideration that is entailed by other accepted propositions must also be accepted (List and Pettit 2002).

In this paper, we continue this line of research and investigate judgment aggregation without the completeness requirement. We drop this requirement, first at the collective level and later at the individual one, replacing it with the weaker requirement of merely deductively closed judgments, not even demanding consistency. Under standard conditions on the aggregation function and tight conditions on the agenda of propositions under consideration, we characterize (strong) oligarchies: ${ }^{3}$ under such an aggregation function, there is a subgroup of individuals - the oligarchs - whose joint acceptance of any proposition is necessary and sufficient for its collective acceptance. Thus the set of collectively accepted propositions is simply the set of propositions unanimously accepted by the oligarchs. Our main result also implies a characterization of the unanimity function, ${ }^{4}$ which is the only anonymous oligarchy. Further corollaries are new variants of existing characterizations of dictatorships (but using no consistency condition). We provide illustrative applications of our results to Arrowian preference aggregation problems and Kasher and Rubinstein's group identification problem.

Our results strengthen Gärdenfors's results in three respects. First, they impose weaker conditions on aggregation functions. Second, they show that strong and not merely weak oligarchies are implied by these conditions and in fact fully characterize them. Third, they do not require the logically rich and infinite agenda of propositions Gärdenfors assumes. They reinforce Gärdenfors's arguments, however, in showing that, under surprisingly mild conditions, we are restricted to oligarchic aggregation functions.

In judgment aggregation, one can distinguish between impossibility results (like the results in List and Pettit 2002, Pauly and van Hees 2006, Dietrich 2006, Gärdenfors 2006) and characterizations of impossibility agendas (like the present results and the results cited below). The former show that, for certain agendas of propositions, aggregation in accordance with certain conditions is impossible or severely restricted (e.g., to dictatorships or oligarchies). The latter characterize the precise class of agendas for which such an impossibility or restriction arises (and thereby also the class of agendas for which it does not arise). Characterizations of impossibility agendas have the merit of identifying precisely which kinds of decision problems are subject to the impossibility results in question and which are free from them. Notoriously, preference aggregation problems are subject to most such impossibility results. There has been much recent progress on such characterizations. Nehring and Puppe (2002) were the

\footnotetext{
${ }^{3}$ These oligarchies have no default. For truth-functional agendas, Nehring and Puppe (2005) have characterized oligarchies with a default, which are distinct from the (strong or weak) oligarchies considered by Gärdenfors (2006) and us. Oligarchies with a default by definition generate complete collective judgments.

${ }^{4}$ Again without a default, thus with possibly incomplete outcomes.
} 
first to prove such results (originally in the context of the theory of strategyproof social choice on generalized single-peaked domains). Subsequent results have been derived by Dokow and Holzman (2005), Dietrich (2007) and Dietrich and List (2007a). But so far all characterizations of impossibility agendas assume fully rational collective judgments. We here give the first characterizations of impossibility agendas without requiring complete or even consistent collective judgments. ${ }^{5}$

\section{The model}

We model a group of individuals who seek to make collective judgments on some propositions. Let us go through the components of our model.

\subsection{Propositions and logic}

We represent propositions as sentences in some logic (Dietrich 2007, generalizing List and Pettit 2002, 2004). The logic is given by:

(i) a set of sentences (called propositions), defined as a set $\mathbf{L} \neq \varnothing$ closed under negation (i.e., if $p \in \mathbf{L}$ then $\neg p \in \mathbf{L}$, with $\neg$ as negation symbol);

and in order to represent logical relationships between propositions:

(ii) an entailment relation, defined as a binary relation $\vDash$ between sets of propositions and propositions, where $A \vDash p$ is read as " $A$ entails $p$ " (with $A \subseteq \mathbf{L}$ and $p \in \mathbf{L}){ }^{6}$

In standard propositional logic, $\mathbf{L}$ contains propositions such as $a, b, a \wedge b$, $a \vee b, \neg(a \rightarrow b)$ (where $\wedge, \vee, \rightarrow$ denote "and", "or", "if-then", respectively) and $\vDash$ satisfies $\{a, a \rightarrow b\} \vDash b,\{a\} \vDash a \vee b$, but not $a \vDash a \wedge b$.

We call a set $A \subseteq \mathbf{L}$ inconsistent if $A \vDash p$ and $A \vDash \neg p$ for some $p \in \mathbf{L}$, and consistent otherwise. The logic is assumed to satisfy four axioms. ${ }^{7}$ Apart from

\footnotetext{
${ }^{5}$ For closely related results, see Dokow and Holzman (2006), as referenced in the acknowledgement note.

${ }^{6}$ Formally, $\models \subseteq \mathcal{P}(\mathbf{L}) \times \mathbf{L}$.

${ }^{7} \mathrm{~L} 1$ (self-entailment): For any $p \in \mathbf{L},\{p\} \vDash p$. L2 (monotonicity): For any $p \in \mathbf{L}$ and any $A \subseteq B \subseteq \mathbf{L}$, if $A \vDash p$ then $B \vDash p$. L3 (completability): $\emptyset$ is consistent, and each consistent set $A \subseteq \mathbf{L}$ has a consistent superset $B \subseteq \mathbf{L}$ containing a member of each pair $p, \neg p \in \mathbf{L}$. L4 (non-paraconsistency): For any $A \subseteq \mathbf{L}$ and any $p \in \mathbf{L}$, if $A \cup\{\neg p\}$ is inconsistent then $A \vDash p$. In L4, the converse implication also holds given L1-L3. So, under L1-L4, the notions of entailment and inconsistency are interdefinable. It follows that we could alternatively use as the primitive notion the system $\mathcal{I}$ of inconsistent sets $Y \subseteq \mathbf{L}$ rather than the entailment relation $\vDash$. The axioms are then: L1*: Every pair $\{p, \neg p\} \subseteq \mathbf{L}$ is inconsistent. L2*: Supersets of inconsistent sets are inconsistent. L3*: Identical to L3. Then we define entailment by $A \vDash p \Leftrightarrow A \cup\{\neg p\} \in \mathcal{I}$ for all $p \in \mathbf{L}$ and $A \subseteq \mathbf{L}$ (so that L4 holds by definition). Under the axioms, using $\vDash$ or $\mathcal{I}$ as the primitive notion is equivalent; the former is more common in logic. For more details, see Dietrich (forthcoming).
} 
standard propositional logic, examples of such logics include predicate, modal and conditional logics.

We call a proposition $p \in \mathbf{L}$ a tautology if $\{\neg p\}$ is inconsistent, a contradiction if $\{p\}$ is inconsistent, and contingent if it is neither a tautology nor a contradiction.

\subsection{The agenda}

The set of propositions on which judgments are to be made is called the agenda. Formally, it is a non-empty subset $X \subseteq \mathbf{L}$ expressible as $X=\left\{p, \neg p: p \in X^{*}\right\}$ for a set $X^{*} \subseteq \mathrm{L}$ of unnegated propositions. For simplicity, double negations in the agenda cancel each other out, i.e., $\neg \neg p$ stands for $p .^{8}$ In our introductory example, $X$ is $\{a, \neg a, b, \neg b, a \wedge b, \neg(a \wedge b)\}$ in standard propositional logic. Informally, the agenda captures a particular decision problem; the generality of the logical framework allows the representation of various realistic decision problems.

\subsection{Individuals and their judgments}

The individuals are represented by the set $N=\{1,2, \ldots, n\}(n \geq 2)$. Each individual $i$ 's judgment set is the set $A_{i} \subseteq X$ of propositions that individual $i$ accepts. On the standard interpretation, to accept a proposition means to believe it (but it could alternatively mean to desire it). A profile is an $n$-tuple $\left(A_{1}, \ldots, A_{n}\right)$ of individual judgment sets. A judgment set $A_{i} \subseteq X$ is

- consistent if it is a consistent set in $\mathbf{L}$,

- complete if, for every proposition $p \in X, p \in A_{i}$ or $\neg p \in A_{i}$,

- deductively closed if, for every proposition $p \in X$, if $A_{i} \vDash p$ then $p \in A_{i}$.

The conjunction of consistency and completeness ("full rationality") implies deductive closure (List 2004, Dietrich 2007). Deductive closure is a much weaker requirement than full rationality; it can be met by small, even empty, judgment sets.

\subsection{Aggregation functions}

A (judgment) aggregation function is a function $F$ that assigns to each admissible profile $\left(A_{1}, \ldots, A_{n}\right)$ a collective judgment set $F\left(A_{1}, \ldots, A_{n}\right)=A \subseteq X$, defined analogously to an individual one and interpreted as the set of propositions that the group accepts. The set of admissible profiles (the domain) is denoted $\operatorname{Dom}(F)$. We call $F$

\footnotetext{
${ }^{8}$ To be precise, when we use the negation symbol $\neg$ hereafter, we mean a modified negation symbol $\sim$, where $\sim p:=\neg p$ if $p$ is unnegated and $\sim p:=q$ if $p=\neg q$ for some $q$.
} 
- universal if $\operatorname{Dom}(F)$ is the "universal" domain of all profiles of consistent and complete individual judgment sets;

- consistent, complete, deductively closed if it generates, respectively, a consistent, complete, deductively closed collective judgment set $F\left(A_{1}, \ldots, A_{n}\right)$ for every profile $\left(A_{1}, \ldots, A_{n}\right) \in \operatorname{Dom}(F)$.

Examples of aggregation functions are majority voting, where, for each $\left(A_{1}, \ldots, A_{n}\right)$ in the universal domain,

$$
F\left(A_{1}, \ldots, A_{n}\right)=\left\{p \in X:\left|\left\{i \in N: p \in A_{i}\right\}\right|>\left|\left\{i \in N: p \notin A_{i}\right\}\right|\right\}
$$

and a dictatorship of individual $i \in N$, where, for each $\left(A_{1}, \ldots, A_{n}\right)$ in the universal domain,

$$
F\left(A_{1}, \ldots, A_{n}\right)=A_{i} .
$$

As the "discursive paradox" shows, majority voting, while universal, is neither consistent nor deductively closed; it is complete when $n$ is odd. Dictatorships are consistent, complete and deductively closed (as well as universal).

\section{Main results}

As noted above, standard impossibility results on judgment aggregation establish the difficulty of finding appealing aggregation functions if we demand consistent and complete collective judgment sets. Are there any appealing aggregation functions if we weaken this requirement to deductive closure alone and thus allow incomplete outcomes? The answer to this question depends on two factors: first, the conditions we impose on the aggregation function (in addition to universality and deductive closure), and second, the richness of the logical interconnections between the propositions in the agenda.

\subsection{Conditions on an aggregation function}

Our results share with previous results the requirement of propositionwise aggregation: the group "votes" independently on each proposition, as captured by the following condition.

Independence. For any $p \in X$ and any $\left(A_{1}, \ldots, A_{n}\right),\left(A_{1}^{*}, \ldots, A_{n}^{*}\right)$ $\in \operatorname{Dom}(F)$, if [for all $i \in N, p \in A_{i} \Leftrightarrow p \in A_{i}^{*}$ ] then $p \in F\left(A_{1}, \ldots, A_{n}\right) \Leftrightarrow$ $p \in F\left(A_{1}^{*}, \ldots, A_{n}^{*}\right)$.

Interpretationally, independence requires the group judgment on any given proposition $p \in X$ to "supervene" on the individual judgments on $p$ (List and Pettit 2006). This reflects a "local" notion of democracy, which could for instance be viewed as underlying direct democratic systems that are based on referenda on various propositions. If we require the group not only to vote 
independently on the propositions, but also to use the same voting method for each proposition (a neutrality condition), we obtain the following stronger condition, required by some of our results.

Systematicity. For any $p, q \in X$ and any $\left(A_{1}, \ldots, A_{n}\right),\left(A_{1}^{*}, \ldots, A_{n}^{*}\right)$ $\in \operatorname{Dom}(F)$, if [for all $i \in N, p \in A_{i} \Leftrightarrow q \in A_{i}^{*}$ ] then $p \in F\left(A_{1}, \ldots, A_{n}\right) \Leftrightarrow$ $q \in F\left(A_{1}^{*}, \ldots, A_{n}^{*}\right)$.

Some of our results also require the following responsiveness condition.

Monotonicity. For any $\left(A_{1}, \ldots, A_{n}\right) \in \operatorname{Dom}(F)$, we have $F\left(A_{1}^{*}, \ldots, A_{n}^{*}\right)=$ $F\left(A_{1}, \ldots, A_{n}\right)$ for all $\left(A_{1}^{*}, \ldots, A_{n}^{*}\right) \in \operatorname{Dom}(F)$ arising from $\left(A_{1}, \ldots, A_{n}\right)$ by replacing one $A_{i}$ by $F\left(A_{1}, \ldots, A_{n}\right)$.

Monotonicity states that changing one individual's judgment set towards the present outcome (collective judgment set) does not alter the outcome. ${ }^{9}$ Finally, throughout the paper, we use the very weak condition of unanimity preservation.

Unanimity preservation. For any unanimous $(A, \ldots, A) \in \operatorname{Dom}(F)$, we have $F(A, \ldots, A)=A$.

\subsection{Agenda richness}

Interesting impossibility results on judgment aggregation never apply to all agendas $X$. Typically, impossibilities using the strong condition of systematicity apply to most agendas of interest, while impossibilities using the weaker condition of independence apply to a class of agendas that both includes and excludes many interesting agendas. Our present results confirm this picture. We use three agenda conditions: two relatively undemanding ones for the systematicity result and one more demanding one for the independence result.

The first two conditions allow a simpler expression for a finite agenda or compact $^{10}$ logic. For expositional ease, we therefore state their simplified form for the finite or compact case here. The general form, valid in all cases, is stated in the appendix. All our results hold, and are proved, in full generality. Call a set of propositions $Y$ minimal inconsistent if it is inconsistent and every proper subset $Z \subsetneq Y$ is consistent. So here are the first two conditions in the finite or compact case:

\footnotetext{
${ }^{9}$ This is a judgment-set-wise monotonicity condition, which differs from a propositionwise one (e.g., Dietrich and List 2005). Similarly, our condition of unanimity-preservation is judgment-set-wise rather than proposition-wise. One may consider this as an advantage, since a flavour of independence is avoided, so that the conditions in the characterization are in the inutitive sense "orthogonal" to each other.

${ }^{10} \mathrm{~A} \operatorname{logic}(\mathbf{L}, \vDash)$ is compact if, for all $A \subseteq \mathbf{L}$ and $p \in \mathbf{L}, A \vDash p$ implies that $B \vDash p$ for some finite subset $B \subseteq A$. Equivalently, given L1-L4, $(\mathbf{L}, \vDash)$ is compact if every inconsistent set $A \subseteq \mathbf{L}$ has a finite inconsistent subset $B \subseteq A$.
} 
(i) There is a minimal inconsistent set $Y \subseteq X$ with $|Y| \geq 3$ (a standard condition with a precursor in abstract aggregation theory, the non-median space condition in Nehring and Puppe 2002).

(ii) There is a minimal inconsistent set $Y \subseteq X$ such that $(Y \backslash Z) \cup\{\neg p: p \in Z\}$ is consistent for some subset $Z \subseteq Y$ of even size (even-number negation condition in Dietrich 2007 and Dietrich and List 2007a; equivalent, for finite $X$, to Dokow and Holzman's 2005 non-affineness).

For any $p, q \in X$, we write $p \vDash^{*} q$ ( $p$ conditionally entails $q$ ) if $\{p\} \cup Y \vDash q$ for some $Y \subseteq X$ consistent with $p$ and with $\neg q$. For instance, for the agenda $X=\{a, \neg a, b, \neg b, a \wedge b, \neg(a \wedge b)\}$, we have $a \wedge b \vDash^{*} a($ take $Y=\emptyset)$ and $a \vDash^{*} \neg b$ (take $Y=\{\neg(a \wedge b)\})$.

(iii) For every contingent $p, q \in X$, there exist $p_{1}, p_{2}, \ldots, p_{k} \in X$ (with $p=$ $p_{1}$ and $q=p_{k}$ ) such that $p_{1} \vDash^{*} p_{2}, p_{2} \vDash^{*} p_{3}, \ldots, p_{k-1} \vDash^{*} p_{k}$ (pathconnectedness, a close variant of Nehring and Puppe's 2002 total blockedness, equivalent for finite $X$ ).

Conditions (i) and (ii) are relatively undemanding; they are met by most standard agendas in the judgment aggregation literature. For instance, if $X$ contains propositions $a, b, a \wedge b$ as in the example of Table 1, then in (i) and (ii) we can take $Y=\{a, b, \neg(a \wedge b)\}$, where in (ii) $Z=\{a, b\}$. If $X$ contains propositions $a, a \rightarrow b, b$, then in (i) and (ii) we can take $Y=\{a, a \rightarrow b, \neg b\}$, where in (ii) $Z=\{a, \neg b\}$. In Sections 4 and 5, we show that the conditions are also met by agendas representing Arrowian preference aggregation or Kasher and Rubinstein's group identification problem. Condition (iii) is more demanding. The agenda $X=\{a, \neg a, b, \neg b, a \wedge b, \neg(a \wedge b)\}$, for example, violates it: for a negated proposition $(\neg a$ or $\neg b$ or $\neg(a \wedge b)$ ), there is no path of pairwise conditional entailments to a non-negated one. The agendas for representing preference aggregation or group identification problems, however, satisfy (iii), as discussed in Sections 4 and 5.

\subsection{Results}

We call an aggregation function $F$ a (strong) oligarchy (dropping "strong" whenever there is no ambiguity) if it is universal and given by

$$
F\left(A_{1}, . ., A_{n}\right)=\cap_{i \in M} A_{i} \text { for each profile }\left(A_{1}, \ldots, A_{n}\right),
$$

where $M \subseteq N$ is a fixed non-empty set (of oligarchs). A weak oligarchy is a universal aggregation function $F$ such that, among all non-empty sets $M \subseteq N$

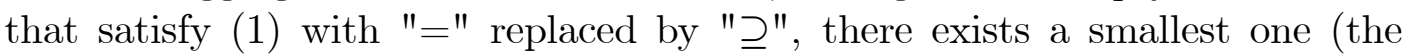
set of weak oligarchs). ${ }^{11}$ An oligarchy (respectively, weak oligarchy) accepts all

\footnotetext{
${ }^{11}$ The term "oligarchy" (without further qualification) refers to a strong oligarchy, whereas in Gärdenfors (2006) it refers to a weak one. A distinct oligarchy notion is Nehring and Puppe's (2005) "oligarchy with a default", which always generates complete collective judgment sets by reverting to a default on each pair $p, \neg p \in X$ on which the oligarchs disagree. Thus oligarchies with a default are special complete weak oligarchies.
} 
(respectively, at least all) propositions unanimously accepted by the oligarchs. While an oligarchy is uniquely determined by the set $M$ of oligarchs, a weak oligarchy is not, ${ }^{12}$ because $F\left(A_{1}, . ., A_{n}\right)$ can be any superset of $\cap_{i \in M} A_{i}$. Further, while oligarchies are independent and incomplete (unless there is a single oligarch or no contingent proposition), weak oligarchies need not satisfy independence, and can be complete.

Theorem 1 Let the agenda $X$ satisfy (i) and (ii).

(a) The oligarchies are the only universal, deductively closed, unanimity-preserving and systematic aggregation functions.

(b) Part (a) continues to hold if the agenda condition (ii) is dropped and the aggregation condition of monotonicity is added.

Theorem 2 Let the agenda $X$ satisfy (ii) and (iii).

(a) The oligarchies are the only universal, deductively closed, unanimity-preserving and independent aggregation functions.

(b) Part (a) continues to hold if the agenda condition (ii) is dropped and the aggregation condition of monotonicity is added.

Proofs are given in the Appendix. Theorems 1 and 2 provide four characterizations of oligarchies. They differ in the conditions imposed on aggregation functions and the agendas permitted. Part (a) of Theorem 2 is perhaps the most surprising result, as it characterizes oligarchies on the basis of the logically weakest set of conditions on aggregation functions. We later apply this result to Arrowian preference aggregation problems and Kasher and Rubinstein's group identification problem.

In each characterization, adding the condition of anonymity - i.e., the requirement that $F\left(A_{1}, \ldots, A_{n}\right)=F\left(A_{1}^{*}, \ldots, A_{n}^{*}\right)$ whenever $\left(A_{1}, \ldots, A_{n}\right)$ and $\left(A_{1}^{*}, \ldots, A_{n}^{*}\right)$ are permutations of each other - eliminates all oligarchies except the unanimity function (i.e., the oligarchy with the set of oligarchs $N$ ), and adding the condition of completeness eliminates all oligarchies except dictatorships (i.e., oligarchies with a single oligarch). So we obtain characterizations of the unanimity function and of dictatorships.

Corollary 1 (a) In each part of Theorems 1 and 2, the unanimity function is the only aggregation function satisfying the specified conditions and anonymity.

(b) In each part of Theorems 1 and 2, dictatorships are the only aggregation functions satisfying the specified conditions and completeness.

Note that none of the characterizations of oligarchic, dictatorial or unanimity functions uses a collective consistency condition: consistency follows from the

\footnotetext{
${ }^{12}$ Unless the agenda contains no contingent propositions (i.e., it contains only tautologies and contradictions).
} 
other conditions, as is seen from the consistency of oligarchic, dictatorial or unanimity functions.

As mentioned in the introduction, our results strengthen Gärdenfors's (2006) oligarchy results. We discuss the exact relationship in Section 6 , when we relax the requirement of completeness not only at the collective level but also at the individual one.

Part (b) of Corollary 1 is also related to the characterizations of dictatorships by Nehring and Puppe (2002), Dokow and Holzman (2005), Dietrich (2007) and Dietrich and List (2007a). To be precise, the dictatorship corollaries derived from parts (a) of Theorems 1 and 2 are variants (without a collective consistency condition) of Dokow and Holzman's (2005) and Dietrich and List's (2007a) characterizations of dictatorships. ${ }^{13}$ The dictatorship corollaries derived from parts (b) of Theorems 1 and 2 are variants (again without a collective consistency condition) of Nehring and Puppe's (2002) characterizations of dictatorships.

As announced in the introduction, we seek to characterize impossibility agendas. While Theorems 1 and 2 establish the sufficiency of our agenda conditions for the present oligarchy results, we also need to establish their necessity. This is done by the next result. The proof consists in the construction of appropriate non-oligarchic counterexamples, given in the Appendix. ${ }^{14}$

Theorem 3 Suppose $n \geq 3$ (and $X$ contains at least one contingent proposition).

(a) If the agenda condition (i) is violated, there is a non-oligarchic aggregation function that is universal, deductively closed, unanimity-preserving, systematic and monotonic.

(b) If the agenda condition (ii) is violated, there is a non-oligarchic (in fact, non-monotonic) aggregation function that is universal, deductively closed, unanimity-preserving and systematic.

(c) If the agenda condition (iii) is violated, and the agenda is finite or belongs to a compact logic, there is a non-oligarchic (in fact, non-systematic) aggregation function that is universal, deductively closed, unanimity-preserving, independent and monotonic.

Each part of theorem 3 continues to hold if consistency is added as a further condition on the aggregation function.

\footnotetext{
${ }^{13}$ For finite $X$, our agenda conditions are equivalent to those of the mentioned other dictatorship characterizations.

${ }^{14}$ Part (c) still holds for $n=2$. It could also be shown using an aggregation function specified by Nehring and Puppe (2002). For finite $X$, part (b) could also be shown using Dokow and Holzman's (2005) parity rule.
} 


\section{Application I: preference aggregation}

We apply Theorem 2 to the aggregation of (strict) preferences, specifically to the case where a profile of fully rational individual preference orderings is to be aggregated into a possibly partial collective preference ordering.

To represent this aggregation problem in the judgment aggregation model, consider the preference agenda (Dietrich and List 2007a; see also List and Pettit 2004), defined as $X=\{x P y, \neg x P y \in \mathbf{L}: x, y \in K$ with $x \neq y\}$, where

(i) $\mathbf{L}$ is a simple predicate logic, with

- a two-place predicate $P$ (representing strict preference), and

- a set of constants $K=\{x, y, z, \ldots\}$ (representing alternatives);

(ii) for each $S \subseteq \mathbf{L}$ and each $p \in \mathbf{L}, S \vDash p$ if and only if $S \cup Z$ entails $p$ in the standard sense of predicate logic, with $Z$ defined as the set of rationality conditions on strict preferences. ${ }^{15}$

We claim that strict preference orderings can be formally represented as judgments on the preference agenda. Call a binary preference relation $\succ$ on $K$ a strict partial ordering if it is asymmetric and transitive, and call $\succ$ a strict ordering if it is in addition connected. Notice that (i) the mapping that assigns to each strict partial ordering $\succ$ the judgment set $A=\left\{x P y, \neg y P x \in X: x \succ_{i}\right.$ $y\} \subseteq X$ is a bijection between the set of all strict partial orderings and the set of all consistent and deductively closed (but not necessarily complete) judgment sets; and (ii) the restriction of this mapping to strict orderings is a bijection between the set of all strict orderings and the set of all consistent and complete (hence deductively closed) judgment sets.

To apply Theorem 2, we observe that the preference agenda for three or more alternatives satisfies the agenda conditions of Theorem 2 .

Lemma 1 If $|K| \geq 3$, the preference agenda satisfies (ii) and (iii).

Proof. Let $X$ be the preference agenda with $|K| \geq 3$. To see that $X$ satisfies (ii) (using the simplified form), take $Y=\{x P y, y P z, z P x\}$ (for distinct alternatives $x, y, z \in K)$, and $Z=\{x P y, y P z\}$. It is shown in Dietrich and List (2007a) that $X$ satisfies (iii) (Nehring 2003 has proved this result for the weak preference agenda).

Corollary 2 For a preference agenda with $|K| \geq 3$, the oligarchies are the only universal, deductively closed (and also consistent), unanimity-preserving and independent aggregation functions.

We have bracketed consistency since the result does not need the condition, although the interpretation offered above assumes it. In the terminology of

\footnotetext{
${ }^{15} Z$ contains $\left(\forall v_{1}\right)\left(\forall v_{2}\right)\left(v_{1} P v_{2} \rightarrow \neg v_{2} P v_{1}\right)$ (asymmetry), $\left(\forall v_{1}\right)\left(\forall v_{2}\right)\left(\forall v_{3}\right)\left(\left(v_{1} P v_{2} \wedge\right.\right.$ $\left.\left.v_{2} P v_{3}\right) \rightarrow v_{1} P v_{3}\right)$ (transitivity), $\left(\forall v_{1}\right)\left(\forall v_{2}\right)\left(\neg v_{1}=v_{2} \rightarrow\left(v_{1} P v_{2} \vee v_{2} P v_{1}\right)\right)$ (connectedness) and, for each pair of distinct contants $x, y \in K, \neg x=y$.
} 
preference aggregation, Corollary 2 shows that the oligarchies are the only preference aggregation functions with universal domain (of strict orderings) generating strict partial orderings and satisfying the weak Pareto principle and independence of irrelevant alternatives. Here an oligarchy is a preference aggregation function such that, for each profile of strict orderings $\left(\succ_{1}, \ldots, \succ_{n}\right)$, the collective strict partial ordering $\succ$ is defined as follows: for any alternatives $x, y \in K, x \succ y$ if and only if $x \succ_{i} y$ for all $i \in M$, where $M \subseteq N$ is an antecedently fixed non-empty set of oligarchs.

This corollary is closely related to Gibbard's (1969) classic result showing that, if the requirement of transitive social orderings in Arrow's framework is weakened to that of quasi-transitive ones (requiring transitivity only for the strong component of the social ordering, but not for the indifference component), then oligarchies (suitably defined for the case of weak preference orderings) are the only preference aggregation functions satisfying the remaining conditions of Arrow's theorem. The relationship to our result lies in the fact that the strong component of a quasi-transitive social ordering is a strict partial ordering, as defined above.

\section{Application II: group identification}

Here we apply Theorem 2 to Kasher and Rubinstein's (1997) problem of "group identification" (see also Samet and Schmeidler 2003). A set $N=\{1,2, \ldots, n\}$ of individuals (e.g., a population) each make a judgment $J_{i} \subseteq N$ on which individuals in that set belong to a particular social group, subject to the constraint that at least one individual belongs to the group but not all individuals do (formally, each $J_{i}$ satisfies $\varnothing \subsetneq J_{i} \subsetneq N$ ). ${ }^{16}$ The individuals then seek to aggregate their judgments $\left(J_{1}, \ldots, J_{n}\right)$ on who belongs to the social group into a resulting collective judgment $J$, subject to the same constraint $(\varnothing \subsetneq J \subsetneq N)$. Thus Kasher and Rubinstein analyse the case in which the group membership status of all individuals must be settled definitively.

By contrast, we apply Theorem 2 to the case in which the membership status of individuals can be left undecided: i.e., some individuals are deemed members of the group in question, others are deemed non-members, and still others are left undecided with regard to group membership, subject to the very minimal "deductive closure" constraint that if all individuals except one are deemed non-members, then the remaining individual must be deemed a member, and if all individuals except one are deemed members, then the remaining individual must be deemed a non-member.

\footnotetext{
${ }^{16}$ This constraint can be interpreted as the conjunction of two feasibility constraints: it is not feasible for the social group in question to be empty, and it is not feasible for it to be universal. This may be plausible if citizenship of a country or membership of a club is at issue. We make some remarks below on what happens if we relax one of these two feasibility constraints.
} 
To represent this problem in our model (drawing on a construction in List forthcoming), consider the group identification agenda, defined as $X=$ $\left\{a_{1}, \neg a_{1}, \ldots, a_{n}, \neg a_{n}\right\}$, where

(i) $\mathbf{L}$ is a simple propositional logic, with atomic propositions $a_{1}, \ldots, a_{n}$ and the standard connectives $\neg, \wedge, \vee$;

(ii) for each $S \subseteq \mathbf{L}$ and each $p \in \mathbf{L}, S \vDash p$ if and only if $S \cup Z$ entails $p$ in the the standard sense of propositional logic, where $Z=\left\{a_{1} \vee \ldots \vee a_{n}, \neg\left(a_{1} \wedge\right.\right.$ $\left.\left.\ldots \wedge a_{n}\right)\right\}$.

Informally, $a_{j}$ is the proposition that "individual $j$ is a member of the social group", and $S \models p$ means that $S$ implies $p$ relative to the constraint that the disjunction of $a_{1}, \ldots, a_{n}$ is true and their conjunction false. The mapping that assigns to each $J$ (with $\varnothing \subsetneq J \subsetneq N$ ) the judgment set $A=\left\{a_{j}: j \in\right.$ $J\} \cup\left\{\neg a_{j}: j \notin J\right\} \subseteq X$ is a bijection between the set of all fully rational judgments in the Kasher and Rubinstein sense and the set of all consistent and complete judgment sets in our model. A merely deductively closed judgment set $A \subseteq X$ represents a judgment that possibly leaves the membership status of some individuals undecided, as outlined above and illustrated more precisely below.

To apply Theorem 2, we observe that the group identification agenda for three or more individuals $(n \geq 3)$ satisfies the agenda conditions of Theorem 2 .

Lemma 2 If $n \geq 3$, the group identification agenda satisfies (ii) and (iii).

Proof. Let $X$ be the group identification agenda with $n \geq 3$. To see that $X$ satisfies (ii) (using the simplified form), take $Y=\left\{a_{j}: j \in N\right\}$, and let $Z$ be an arbitrary binary subset of $Y$. It is shown in List (forthcoming) that $X$ satisfies (iii).

Corollary 3 For a group identification agenda with $n \geq 3$, the oligarchies are the only universal, deductively closed (and consistent), unanimity-preserving and independent aggregation functions.

In group identification terms, the oligarchies are the only group identification functions with universal domain generating possibly incomplete but deductively closed group membership judgments and satisfying unanimity and independence. ${ }^{17}$ Here an oligarchy is a group identification function such that, for each profile $\left(J_{1}, \ldots, J_{n}\right)$ of fully rational individual judgments on group membership,

\footnotetext{
${ }^{17}$ If the feasibility constraint on membership is relaxed so as to allow either an empty group or a universal one, then other group identification functions satisfying the conditions become available: for example, oligarchies with the default of non-membership (if an empty group is allowed) or with the default of membership (if a universal group is allowed). However, our systematicity result (part (a) of theorem 1) continues to apply under the weakened feasibility constraints.
} 
the collective judgment is given as follows: the set of determinate group members is $\bigcap_{i \in M} J_{i}$, the set of determinate non-members is $\bigcap_{i \in M}\left(N \backslash J_{i}\right)$, and the set of individuals with undecided membership status is the complement of these two sets in $N$, where $M \subseteq N$ is an antecedently fixed non-empty set of oligarchs. ${ }^{18}$

\section{The case of incomplete individual judgments}

As argued by Gärdenfors (2006), it is natural to relax the completeness requirement not only at the collective level, but also at the individual one. Do the above impossibilities disappear if individuals can withhold judgments on some or even all pairs $p, \neg p \in X$ ? The answer to this question is negative, even if the conditions of independence or systematicity are weakened by allowing the collective judgment on a proposition $p \in X$ to depend not only on the individuals' judgments on $p$ but also on those on $\neg p$. Such weaker independence or systematicity conditions are arguably more defensible than the standard conditions: $\neg p$ is intimately related to $p$, and thus individual judgments on $\neg p$ should be allowed to matter for group judgments on $p$. As the weakened conditions are equivalent to the standard ones under individual completeness, all the results in Section 3 continue to hold for the weakened independence and systematicity conditions.

Formally, call $F$ universal ${ }^{*}$ if $\operatorname{Dom}(F)$ is the "extended" universal domain of all profiles of consistent and deductively closed (but not necessarily complete) individual judgment sets (this is a superdomain of the original universal domain). An oligarchy* is the universal* variant of an oligarchy as defined above. Following Gärdenfors (2006), call $F$ weakly independent if, for any $p \in X$ and any $\left(A_{1}, \ldots, A_{n}\right),\left(A_{1}^{*}, \ldots, A_{n}^{*}\right) \in \operatorname{Dom}(F)$, if [for all $i \in N, p \in A_{i} \Leftrightarrow p \in A_{i}^{*}$ and $\left.\neg p \in A_{i} \Leftrightarrow \neg p \in A_{i}^{*}\right]$ then $p \in F\left(A_{1}, \ldots, A_{n}\right) \Leftrightarrow p \in F\left(A_{1}^{*}, \ldots, A_{n}^{*}\right)$. Likewise, call $F$ weakly systematic if, for any $p, q \in X$ and any $\left(A_{1}, \ldots, A_{n}\right),\left(A_{1}^{*}, \ldots, A_{n}^{*}\right) \in$ $\operatorname{Dom}(F)$, if [for all $i \in N, p \in A_{i} \Leftrightarrow q \in A_{i}^{*}$ and $\neg p \in A_{i} \Leftrightarrow \neg q \in A_{i}^{*}$ ] then $p \in F\left(A_{1}, \ldots, A_{n}\right) \Leftrightarrow q \in F\left(A_{1}^{*}, \ldots, A_{n}^{*}\right)$.

We now give analogues of parts (a) of Theorems 1 and 2, proved in the Appendix.

Theorem 1* Let the agenda X satisfy (i) and (ii). The oligarchies* are the only universal ${ }^{*}$, deductively closed, unanimity-preserving and weakly systematic aggregation functions.

\footnotetext{
${ }^{18}$ The set of individuals whose group membership status is to be decided need not coincide with the set of individuals who submit judgments on who is a member. More generally, the set $N$ can make judgments on which individuals in another set $K(|K| \geq 3)$ belong to a particular social group, subject to the constraint stated above. $K$ can be infinite. Corollary 3 continues to hold since the corresponding group identification agenda (for a suitably adapted logic) still satisfies (ii) and (iii). Interestingly, if $K$ is infinite the agenda belongs to a non-compact logic.
} 
Theorem 2* Let the agenda X satisfy (ii) and (iii). The oligarchies* are the only universal*, deductively closed, unanimity-preserving and weakly independent aggregation functions.

In analogy with Theorems 1 and 2, these characterizations of oligarchies* do not contain a collective consistency condition (but require individual consistency). In each of Theorems $1^{*}$ and $2^{*}$, adding the collective completeness requirement (respectively, anonymity) narrows down the class of aggregation functions to dictatorial ones (respectively, the unanimity function), extended to the domain of all profiles of consistent and deductively closed individual judgment sets. So Theorems $1^{*}$ and $2^{*}$ imply characterizations of the latter functions on the extended universal domain. Further, our applications of Theorem 2 to the preference and group identification agendas in Sections 4 and 5 can accommodate the case of incomplete individual judgments by using Theorem $2^{*}$ instead of Theorem 2.

We can finally revisit the relationship of our results with Gärdenfors's results. Theorem 2, Corollary 1 and Theorem $2^{*}$ strengthen Gärdenfors's oligarchy results. First, they do not require Gärdenfors's "social consistency" condition. ${ }^{19}$ Second, they show that the conditions on aggregation functions imply (and in fact fully characterize) strong and not merely weak oligarchies (respectively, oligarchies*). Third, they weaken Gärdenfors's assumption that the agenda has the structure of an atomless Boolean algebra, replacing it with the weakest possible agenda condition under which the oligarchy result holds, i.e., (ii) and (iii). ${ }^{20}$

Our results reinforce the observation that, if we seek to avoid the standard impossibility results on judgment aggregation by allowing incomplete judgments while preserving the requirements of deductive closure and (weak) independence, this route does not lead very far. To obtain genuine possibilities, deductive closure must be relaxed or - perhaps better - independence must be given up in favour of non-propositionwise aggregation functions.

\section{References}

Alchourron CE, Gärdenfors P, Makinson D (1985) On the logic of theory change: partial meet contraction and revision functions. Journal of Symbolic Logic 50: 510-530

\footnotetext{
${ }^{19}$ Gärdenfors's "social logical closure" is equivalent to our "deductive closure", where entailment in Gärdenfors' Boolean algebra agenda $X$ should be defined as follows: a set $A \subseteq X$ entails $p \in X$ if and only if $\left(\wedge_{q \in A_{0}} q\right) \wedge \neg p$ is the contradiction for some finite $A_{0} \subseteq A$.

${ }^{20}$ It is easily checked that Gärdenfors's agenda satisfies (ii) and (iii), where paths involving at most two conditional entailments exist between any two propositions. To be precise, our present generalization of Gärdenfors's Corollary 3 applies to the case of a finite number of individuals. A similar generalization can be given for the infinite case.
} 
Dietrich F (2006) Judgment Aggregation: (Im)Possibility Theorems. Journal of Economic Theory 126(1): 286-298

Dietrich F (2007) A generalised model of judgment aggregation. Social Choice and Welfare 28(4): 529-565

Dietrich F, List C (2007a) Arrow's theorem in judgment aggregation. Social Choice and Welfare 29(1): 19-33

Dietrich F, List C (2007b) Judgment aggregation by quota rules: majority voting generalized. Journal of Theoretical Politics 19(4) (in press)

Dietrich F, List C (forthcoming) Strategy-proof judgment aggregation. Economics and Philosophy

Dokow E, Holzman R (2005) Aggregation of binary evaluations. Working paper, Technion Israel Institute of Technology

Dokow E, Holzman R (2006) Aggregation of binary evaluations with abstentions. Working paper, Technion Israel Institute of Technology

Gärdenfors P (2006) An Arrow-like theorem for voting with logical consequences. Economics and Philosophy 22(2): 181-190

Kasher A, Rubinstein A (1997) On the Question "Who is a J?": A Social Choice Approach. Logique et Analyse 160: 385-395

Konieczny S, Pino-Perez R (2002) Merging information under constraints: a logical framework. Journal of Logic and Computation 12: 773-808

Kornhauser L A, Sager LG (1986) Unpacking the Court. Yale Law Journal 96(1): $82-117$

List C (2004) A Model of Path-Dependence in Decisions over Multiple Propositions. American Political Science Review 98(3): 495-513

List $\mathrm{C}$ (forthcoming) Which worlds are possible? A judgment aggregation problem. Journal of Philosophical Logic

List C, Pettit P (2002) Aggregating Sets of Judgments: An Impossibility Result. Economics and Philosophy 18: 89-110

List C, Pettit P (2004) Aggregating Sets of Judgments: Two Impossibility Results Compared. Synthese 140(1-2): 207-235

List C, Pettit P (2006) Group Agency and Supervenience. Southern Journal of Philosophy XLIV (Spindel Supplement): 85-105

Mongin P (2005) Factoring out the impossibility of logical aggregation. Working paper, CNRS, Paris

Nehring K (2003) Arrow's theorem as a corollary. Economics Letters 80(3): 379-382

Nehring K, Puppe C (2002) Strategy-Proof Social Choice on Single-Peaked Domains: Possibility, Impossibility and the Space Between. Working paper, University of California at Davies

Nehring K, Puppe C (2005) Consistent Judgment Aggregation: A Charac- 
terization. Working paper, University of Karlsruhe

Pauly M, van Hees M (2006) Logical Constraints on Judgment Aggregation. Journal of Philosophical Logic 35: 569-585

Pettit P (2001) Deliberative Democracy and the Discursive Dilemma. Philosophical Issues 11: 268-299

Rubinstein A, Fishburn P (1986) Algebraic Aggregation Theory. Journal of Economic Theory 38: 63-77

Samet D, Schmeidler D (2003) Between liberalism and democracy. Journal of Economic Theory 110: 213-233

van Hees M (2007) The limits of epistemic democracy. Social Choice and Welfare 28(4): 649-666

Wilson R (1975) On the Theory of Aggregation. Journal of Economic Theory 10: $89-99$

\section{A Appendix: proofs}

We first introduce some notation. Let $\mathcal{C}$ be the set of all consistent and complete judgment sets $A \subseteq X$, and $\mathcal{C}^{*}$ the set of all consistent and deductively closed (but not necessarily complete) ones.

For all $B \subseteq X$,

- if $B$ is consistent let $A_{B} \subseteq X$ be any consistent and complete judgment set such that $B \subseteq A_{B}\left(A_{B}\right.$ is a "completion" of $B$ and exists by L1-L3);

- let $\bar{B}:=\{p \in X: B \vDash p\}$ ( $\bar{B}$ is the "deductive closure" of $B)$;

- let $B\urcorner:=\{\neg p: p \in B\}$.

Also, for any sets $Z \subseteq Y$, let $Y_{\neg Z}$ denote the set $(Y \backslash Z) \cup\{\neg p: p \in Z\}$, which arises from $Y$ by negating the propositions in $Z$.

Further, when we consider a profile $\left(A_{1}, \ldots, A_{n}\right)$, we often write $N_{p}$ for the set $\left\{i: p \in A_{i}\right\}$ of individuals accepting $p \in X$.

Finally, for any $\mathcal{W} \subseteq \mathcal{P}(N)$ (which can be arbitrary, even empty), let $F_{\mathcal{W}}$ be the universal aggregation function given by

$$
F\left(A_{1}, \ldots, A_{n}\right)=\left\{p \in X: N_{p} \in \mathcal{W}\right\} \text { for each profile }\left(A_{1}, \ldots, A_{n}\right) \in \mathcal{C}^{n} .
$$

Let us first state conditions (i) and (ii) in full generality.

(i) There is an inconsistent set $Y \subseteq X$ with pairwise disjoint subsets $Z_{1}, Z_{2},\{p\}$ such that $Y_{\neg Z_{1}}, Y_{\neg Z_{2}}$ and $Y_{\neg\{p\}}$ are consistent.

(ii) There is an inconsistent set $Y \subseteq X$ with disjoint subsets $Z,\{p\}$ such that $Y_{\neg Z}, Y_{\neg\{p\}}$ and $Y_{\neg(Z \cup\{p\})}$ are consistent.

The following lemma illuminates their logical relationship with the simplified expressions stated in the main text, which we now relabel ( $\left.i_{\text {main text }}\right)$ and (ii main text), respectively. 
Lemma 3 (a) If $X$ is finite or belongs to a compact logic, (i) is equivalent to $\left(i_{\text {main text }}\right)$, and $(i i)$ is equivalent to (ii $\left.i_{\text {main text }}\right)$.

(b) In general, $\left(i_{\text {main text }}\right)$ implies (i), and (ii $\left.i_{\text {main text }}\right)$ implies (ii).

Proof. To prove (a) and (b), we show the implications (i) $\Rightarrow\left(\mathrm{i}_{\text {main text }}\right)$, $\left(\mathrm{i}_{\text {main text }}\right) \Rightarrow(\mathrm{i}), \quad(\mathrm{ii}) \Rightarrow\left(\mathrm{ii}_{\text {main text }}\right)$, and ( $\left.\mathrm{ii}_{\text {main text }}\right) \Rightarrow(\mathrm{ii})$, where in the first and third implication we assume that $X$ is finite or the logic is compact.

(i) $\Rightarrow\left(\mathrm{i}_{\text {main text }}\right)$. Let $Y, Z_{1}, Z_{2}, p$ be as in (i), and $Y^{\prime} \subseteq Y$ a minimal inconsistent set (which exists by the assumption of a finite $X$ or compact logic). As by (i) $Y^{\prime}$ intersects with each of $Z_{1}, Z_{2},\{p\},\left|Y^{\prime}\right| \geq 3$, implying ( $\mathrm{i}_{\text {main text }}$ ).

$\left(\mathrm{i}_{\text {main text }}\right) \Rightarrow(\mathrm{i})$. Let $Y$ be as in $\left(\mathrm{i}_{\text {main text }}\right)$, and $Z_{1}, Z_{2},\{p\}$ disjoint singleton subsets of $Y$.

(ii) $\Rightarrow\left(\mathrm{ii}_{\text {main text }}\right)$. Let $Y, Z, p$ be as in (ii). ( $\left.\mathrm{ii}_{\text {main text }}\right)$ holds for a minimal inconsistent set $Y^{\prime} \subseteq Y$ (which exists by the assumption of a finite $X$ or compact logic) and the subset $Z \cap Y^{\prime}$ or $\left(Z \cap Y^{\prime}\right) \cup\{p\}$ (whichever has even size).

(ii main text $) \Rightarrow$ (ii). Let $Y$ be as in (ii main text ), and choose a $Z \subseteq Y$ of smallest even size such that $Y_{\neg Z}$ is consistent. If $Y_{\neg Z^{\prime}}$ is consistent for a $Z^{\prime} \subseteq Z$ of size $|Z|-1$, one easily checks that (ii) holds for $Y$ with disjoint subsets $Z^{\prime},\{p\}=$ $Z \backslash Z^{\prime}$. Now assume

$$
Y_{\neg Z^{\prime}} \text { is inconsistent for all } Z^{\prime} \subseteq Z \text { of size }|Z|-1 \text {. }
$$

Then $|Z| \geq 4$, as $|Z|$ is even, not zero (otherwise $Y_{\neg Z}=Y$, which is inconsistent) and not 2 (otherwise, by $Y$ 's minimal inconsistency, $Y_{\neg Z^{\prime}}$ would be consistent for subsets $Z^{\prime} \subseteq Z$ of size $|Z|-1=1$ ). So $Y$ contains no pair $r, \neg r$ (something we will implicitly use), and contains distinct $p, q \in Z$. Let

$$
\widetilde{Z}:=(Z \backslash\{p, q\})\urcorner, Y^{\prime}:=(Y \backslash Z) \cup \widetilde{Z} \cup\{p\} .
$$

We show (ii) for the set $Y^{\prime}$ with disjoint subsets $\{p\}, \widetilde{Z}$.

First, $Y^{\prime}$ is inconsistent as $Y^{\prime} \cup\{q\}$ and $Y^{\prime} \cup\{\neg q\}$ are inconsistent: $Y^{\prime} \cup\{q\}=$ $Y_{\neg(Z \backslash\{p, q\})}$ by $Z^{\prime}$ 's minimality property, and $Y^{\prime} \cup\{\neg q\}=Y_{\neg(Z \backslash\{p\})}$ by (2).

Second, $Y_{\neg \widetilde{Z}}^{\prime}=Y \backslash\{q\}$ and $Y_{\neg(\{p\} \cup \widetilde{Z})}^{\prime}=Y_{\neg\{p\}} \backslash\{q\}$ are consistent by $Y^{\prime}$ 's minimal inconsistency; and $Y_{\neg\{p\}}^{\prime}$ is so by $Y_{\neg\{p\}}^{\prime} \subseteq Y_{\neg Z}$ and $Y_{\neg Z}$ 's consistency.

The next two lemmas have simple proofs, which we leave to the reader.

Lemma 4 The intersection of deductively closed judgment sets is deductively closed. In particular, oligarchies are deductively closed.

Lemma 5 (a) $F$ is universal and systematic if and only if $F=F_{\mathcal{W}}$ for some $\mathcal{W} \subseteq \mathcal{P}(N)$.

(b) $F$ is oligarchic if and only if $F=F_{\{C \subseteq N: M \subseteq C\}}$ for some $\emptyset \neq M \subseteq N$.

(c) Let $X$ contain a contingent proposition. Then, for all $\mathcal{W}, \mathcal{W}^{\prime} \in \mathcal{P}(N)$, 
- if $\mathcal{W} \neq \mathcal{W}^{\prime}$ then $F_{\mathcal{W}} \neq F_{\mathcal{W}^{\prime}}$

- $F_{\mathcal{W}}$ is unanimity-preserving if and only if $N \in \mathcal{W}$ and $\emptyset \notin \mathcal{W}$;

- $F_{\mathcal{W}}$ is monotonic if and only if

$$
C \in \mathcal{W} \& C \subseteq C^{*} \subseteq N \Rightarrow C^{*} \in \mathcal{W}
$$

The next two lemmas are the essential steps towards Theorem 1.

Lemma 6 Let $X$ satisfy (ii). For all $\mathcal{W} \subseteq \mathcal{P}(N)$, if $F_{\mathcal{W}}$ is unanimity-preserving and deductively closed, then $(3)$ holds, i.e. $F_{\mathcal{W}}$ is monotonic by Lemma 5.

Proof. Assume (ii). Let $\mathcal{W} \subseteq \mathcal{P}(N)$, and suppose $F:=F_{\mathcal{W}}$ is unanimitypreserving and deductively closed. We assume $C \in \mathcal{W} \& C \subseteq C^{*} \subseteq N$ and show $C^{*} \in \mathcal{W}$. Let $Y, Z, p$ be as specified in (ii). A profile $\left(A_{1}, \ldots, A_{n}\right)$ can be defined (using the above notation) by

$$
A_{i}= \begin{cases}A_{Y_{\neg\{p\}}} & \text { if } i \in C \\ A_{Y_{\neg Z}} & \text { if } i \in N \backslash C^{*} \\ A_{Y_{\neg(Z \cup\{p\})}} & \text { if } i \in C^{*} \backslash C,\end{cases}
$$

where we used that $Y_{\neg\{p\}}, Y_{\neg Z}$ and $Y_{\neg(Z \cup\{p\})}$ are consistent sets by (ii). Now $F\left(A_{1}, \ldots, A_{n}\right)$ contains all $q \in Z$ by $N_{q}=C \in \mathcal{W}$, and all $q \in Y \backslash(Z \cup\{p\})$ by $N_{q}=N \in \mathcal{W}$. So $Y \backslash\{p\} \subseteq F\left(A_{1}, \ldots, A_{n}\right)$. By $Y$ 's inconsistency (and L4), $Y \backslash\{p\} \vDash \neg p$, whence $F\left(A_{1}, \ldots, A_{n}\right) \vDash \neg p$. So, by deductive closure, $\neg p \in$ $F\left(A_{1}, \ldots, A_{n}\right)$. Hence $N_{\neg p} \in \mathcal{W}$, i.e. $C^{*} \in \mathcal{W}$, as desired.

Lemma 7 Let $X$ satisfy (i). For all $\emptyset \neq \mathcal{W} \subseteq \mathcal{P}(N)$ satisfying $(3), F_{\mathcal{W}}$ is deductively closed if and only if $\mathcal{W}=\{C \subseteq N: M \subseteq C\}$ for some $M \subseteq N$.

Proof. Let $X$ and $\mathcal{W}$ be as specified. Let $F:=F_{\mathcal{W}}$.

First, suppose $\mathcal{W}=\{C \subseteq N: M \subseteq C\}$ for some $M \subseteq N$. If $M \neq \emptyset$, then $F$ is oligarchic by Lemma $5(\mathrm{~b})$, hence deductively closed by Lemma 4 . If $M=\emptyset$, then $\mathcal{W}=\mathcal{P}(N)$ by (3), whence $F$ always generates the full set $X$, hence is again deductively closed.

Second, suppose $F$ is deductively closed. Note that, to show that $\mathcal{W}=$ $\{C \subseteq N: M \subseteq C\}$ for some $M \subseteq N$, it suffices (by $\mathcal{W} \neq \emptyset$ and (3)) to show that $\mathcal{W}$ is closed under taking finite intersections. Let $W, W^{\prime} \in \mathcal{W}$, and let us show that $W \cap W^{\prime} \in \mathcal{W}$. Let $Y, Z_{1}, Z_{2},\{p\}$ be as in (i), and consider the profile $\left(A_{1}, \ldots, A_{n}\right)$ given (in the above notation) by

$$
A_{i}= \begin{cases}A_{Y_{\neg Z_{1}}} & \text { if } i \in N \backslash W \\ A_{Y_{\neg Z_{2}}} & \text { if } i \in W \backslash W^{\prime} \\ A_{Y_{\neg\{p\}}} & \text { if } i \in W \cap W^{\prime},\end{cases}
$$


where we use that $Y_{\neg Z_{1}}, Y_{\neg Z_{2}}$ and $Y_{\neg\{p\}}$ are each consistent by (i). Then $F\left(A_{1}, \ldots, A_{n}\right)$ contains all $q \in Z_{1}$ by $N_{q}=N \backslash(N \backslash W)=W \in \mathcal{W}$, contains all $q \in Z_{2}$ by $N_{q}=N \backslash\left(W \backslash W^{\prime}\right) \supseteq W^{\prime} \in \mathcal{W}$ and (3), and contains all $r \in Y \backslash\left(Z_{1} \cup Z_{2} \cup\{p\}\right)$ by $N_{r}=N \in \mathcal{W}$. So $Y \backslash\{p\} \subseteq F\left(A_{1}, \ldots, A_{n}\right)$. By $Y$ 's inconsistency (and L4), $Y \backslash\{p\} \vDash \neg p$. Hence $F\left(A_{1}, \ldots, A_{n}\right) \vDash \neg p$, so that by deductive closure $\neg p \in F\left(A_{1}, \ldots, A_{n}\right)$. Hence $N_{\neg p} \in \mathcal{W}$, i.e. $W \cap W^{\prime} \in \mathcal{W}$, as desired.

Proof of Theorem 1. We prove first part (b) and then part (a).

(b) Let (i) hold. As noted above, oligarchies satisfy the specified conditions. Now suppose $F$ satisfies the conditions. By Lemma $5(\mathrm{a}), F=F_{\mathcal{W}}$ for some $\mathcal{W} \subseteq \mathcal{P}(N)$, where by Lemma $5(\mathrm{c}) \mathcal{W}$ satisfies $(3), \emptyset \notin \mathcal{W}$ and $N \in \mathcal{W}$. Hence Lemma 7 applies, so that $\mathcal{W}=\{C \subseteq N: M \subseteq C\}$ for some $M \subseteq N$. As $\emptyset \notin \mathcal{W}, M \neq \emptyset$. So, by Lemma $5(\mathrm{~b}), F$ is oligarchic.

(a) Let (i) and (ii) hold. Again, as noted, oligarchies have the specified properties. Suppose now that $F$ has these properties. By Lemma $5(\mathrm{a}), F=F_{\mathcal{W}}$ for some $\mathcal{W} \subseteq \mathcal{P}(N)$. By Lemma $6, F$ is monotonic. So, by part (b), $F$ is oligarchic.

Theorem 2 follows from Theorem 1 with the help of two further lemmas. The first lemma is similar to a proof step in Dietrich and List (forthcoming), and the second lemma shows that a standard argument, first made by Nehring and Puppe (2002), requires neither completeness and consistency, nor monotonicity.

Lemma 8 If $X$ satisfies (iii) and contains a contingent proposition, (i) holds.

Proof. Let $X$ be as specified. Then there are a contingent $q \in X$, and propositions $q=p_{1}, p_{2}, \ldots, p_{k}=\neg q \in X$ such that $p_{t} \vDash^{*} p_{t+1}$ for all $t \in\{1, \ldots, k-$ $1\}$. We first show that $p_{t} \not \forall p_{t+1}$ for some $t \in\{1, \ldots, k-1\}$. Assume the contrary holds. As $\left\{p_{1}\right\}=\{q\}$ is consistent and $p_{1} \vDash p_{2},\left\{p_{1}, p_{2}\right\}$ is consistent. So, as $p_{2} \vDash p_{3},\left\{p_{1}, p_{2}, p_{3}\right\}$ is consistent. Repeating this procedure, $\left\{p_{1}, \ldots, p_{k}\right\}$ is consistent. But then $\left\{p_{1}, p_{k}\right\}=\{q, \neg q\}$ is consistent, a contradiction.

As just shown, there is a $t \in\{1, \ldots, k-1\}$ with $p_{t} \not \models p_{t+1}$. As $p_{t} \vDash^{*} p_{t+1}$, we have $\left\{p_{t}\right\} \cup Y^{*} \vDash p_{t+1}$ for a $Y^{*} \subseteq X$ consistent with each of $p_{t}$ and $\neg p_{t+1}$. It follows that

$$
\begin{aligned}
& \left\{p_{t}, \neg p_{t+1}\right\} \cup Y^{*} \text { is inconsistent, } \\
& \left\{p_{t}, p_{t+1}\right\} \cup Y^{*} \text { and }\left\{\neg p_{t}, \neg p_{t+1}\right\} \cup Y^{*} \text { are each consistent.. }
\end{aligned}
$$

By $p_{t} \not \forall p_{t+1}$, we have $Y^{*} \neq \emptyset$. Since $\left\{p_{t}, \neg p_{t+1}\right\}$ is consistent, $\left\{p_{t}, \neg p_{t+1}\right\} \cup B$ is consistent for some set $B$ consisting of exactly one member of each pair $r, \neg r$ in $\left\{r, \neg r: r \in Y^{*}\right\}$. Now we define $Y:=\left\{p_{t}, \neg p_{t+1}\right\} \cup Y^{*}, p:=p_{t}$, $Z_{1}:=\left\{\neg p_{t+1}\right\}$, and we let $Z_{2}$ be the subset of $Y^{*}$ for which $Y_{\neg Z_{2}}^{*}=B$. Then, as required in (i), $Y=\left\{p_{t}, \neg p_{t+1}\right\} \cup Y^{*}$ is inconsistent (by (4)), and $Z_{1}, Z_{2},\{p\}$ are 
pairwise disjoint subsets of $Y$, where the three sets $Y_{\neg\{p\}}=\left\{\neg p_{t}, \neg p_{t+1}\right\} \cup Y_{t}^{*}$, $Y_{\neg Z_{1}}=\left\{p_{t}, p_{t+1}\right\} \cup Y_{t}^{*}$ and $Y_{\neg Z_{2}}=\left\{p_{t}, \neg p_{t+1}\right\} \cup B$ are consistent (in the first two cases by (5)).

Call $C \subseteq N$ semi-winning for $p \in X$ (under $F$ ) if $p \in F\left(A_{1}, \ldots, A_{n}\right)$ for all profiles $\left(A_{1}, \ldots, A_{n}\right)$ in the domain with $\left\{i: p \in A_{i}\right\}=C$.

Lemma 9 Let $F$ be universal, deductively closed, independent and unanimitypreserving.

(a) For all $p, q \in X$, if $C \subseteq N$ is semi-winning for $p$ and $p \vDash^{*} q$ then $C$ is semi-winning for $q$.

(b) If $X$ satisfies (iii), $F$ is systematic.

Proof. Let $F$ be as specified.

(a) Consider $p, q \in X$. Suppose $C \subseteq N$ is semi-winning for $p$ and $p \vDash^{*} q$. By $p \vDash^{*} q$, there is a $Y \subseteq X$ such that $\{p\} \cup Y \vDash q$, and $\{p\} \cup Y$ and $\{\neg q\} \cup Y$ are consistent. So, as $\{p, \neg q\} \cup Y$ is inconsistent, $\{p, q\} \cup Y$ and $\{\neg p, \neg q\} \cup Y$ are each consistent. Let $\left(A_{1}, \ldots, A_{n}\right)$ be the profile given (in the above notation) by

$$
A_{i}= \begin{cases}A_{\{p, q\} \cup Y} & \text { if } i \in C \\ A_{\{\neg p, \neg q\} \cup Y} & \text { if } i \notin C .\end{cases}
$$

As $N_{p}=C$ and $C$ is semi-winning for $p, p \in F\left(A_{1}, \ldots, A_{n}\right)$. From unanimitypreservation and independence it follows that $Y \subseteq F\left(A_{1}, \ldots, A_{n}\right)$. So $\{p\} \cup Y \subseteq$ $F\left(A_{1}, \ldots, A_{n}\right)$. Hence, by $\{p\} \cup Y \vDash q$ and deductive closure, $q \in F\left(A_{1}, \ldots, A_{n}\right)$. So, by $N_{q}=C$ and independence, $C$ is semi-winning for $q$, as desired.

(b) Let $X$ satisfy (iii). To show systematicity, consider any $p, q \in X$ and any $\left(A_{1}, \ldots, A_{n}\right),\left(A_{1}^{*}, \ldots, A_{n}^{*}\right) \in \mathcal{C}^{n}$ such that $C:=\left\{i: p \in A_{i}\right\}=\{i: q \in$ $\left.A_{i}^{*}\right\}$. We suppose that $p \in F\left(A_{1}, \ldots, A_{n}\right)$ and prove that $q \in F\left(A_{1}^{*}, \ldots, A_{n}^{*}\right)$. The latter holds if $C=N$ : if $C=N$ then, using unanimity-preservation and independence, it follows that $q \in F\left(A_{1}^{*}, \ldots, A_{n}^{*}\right)$, as desired. Now let $C \neq$ $N$. We have $C \neq \emptyset$, because otherwise, again by unanimity-preservation and independence, we have $p \notin F\left(A_{1}, \ldots, A_{n}\right)$, a contradiction. As $C$ is neither $N$ nor $\emptyset, p$ and $q$ are each contingent (by individual rationality). Hence, by (iii), there are $p=p_{1}, p_{2}, \ldots, p_{k}=q \in X$ such that $p_{1} \vDash^{*} p_{2}, p_{2} \vDash^{*} p_{3}, \ldots, p_{k-1} \vDash^{*} p_{k}$. By $C=\left\{i: p \in A_{i}\right\}, p \in F\left(A_{1}, \ldots, A_{n}\right)$ and independence, $C$ is semi-winning for $p=p_{1}$. So a simple induction using part (a) tells us that $C$ is semi-winning for $p_{k}=q$, as desired.

We base come to the proof of Theorems $1^{*}$, which we derive from Theorem 1 using two lemmas.

Lemma 10 For all $A \subseteq X$, the "deductive closure" $\bar{A}(=\{r \in X: A \vDash r\})$ is deductively closed, and it is consistent if $A$ is consistent. 
Proof. Let $A \subseteq X$.

To show that $\bar{A}$ is deductively closed suppose for a contradiction that $r \in X$ with $\bar{A} \vDash r$ but $r \notin \bar{A}$. Then $A \not \forall r$. So, by L4, $\{\neg r\} \cup A$ is consistent, hence extendible to a complete and consistent $B \subseteq X$ with $\{\neg r\} \cup A \subseteq B$. As $B$ is deductively closed, $\bar{A} \subseteq B$. So $\{\neg r\} \cup \bar{A} \subseteq B$. So $\{\neg r\} \cup \bar{A}$ is consistent. Hence $\bar{A} \not \models r$, a contradiction.

Now let $A$ be consistent. Then $A$ is extendible to a complete and consistent set $B \subseteq X$. As $B$ is deductively closed, $\bar{A} \subseteq B$. So $\bar{A}$ is consistent.

For all $C, C^{\prime} \subseteq N$, we call $C$ semi-winning against $C^{\prime}$ for $p \in X$ (under $F)$ if $p \in F\left(A_{1}, \ldots, A_{n}\right)$ for all profiles $\left(A_{1}, \ldots, A_{n}\right)$ in the domain with $\{i: p \in$ $\left.A_{i}\right\}=C$ and $\left\{i: \neg p \in A_{i}\right\}=C^{\prime}$; and we call $C$ simply semi-winning against $C^{\prime}$ (under $F$ ) if $C$ is semi-winning against $C^{\prime}$ for every $p \in X$. Note that a weakly systematic aggregation function $F$ is uniquely given by its set of pairs $\left(C, C^{\prime}\right) \in(\mathcal{P}(N))^{2}$ for which $C$ is semi-winning against $C^{\prime}$.

Lemma 11 Let $F$ be universal*, deductively closed, unanimity-preserving and weakly systematic. Let $C \subseteq N$ be semi-winning against $\widetilde{C} \subseteq N$, with $C \cap \widetilde{C}=\emptyset$.

(a) If $X$ satisfies (ii), $C$ is semi-winning against all $C^{\prime} \subseteq \widetilde{C}$.

(b) If $X$ satisfies ( $i$ ) and (ii), $C$ is semi-winning against all $C^{\prime} \subseteq N$, i.e. is semi-winning.

Proof. Let $X, F, C, \widetilde{C}$ be as specified.

(a) Assume (ii) holds, and consider any $C^{\prime} \subseteq \widetilde{C}$. By (ii) there are pairwise disjoint sets $Y^{*}, Z,\{p\} \subseteq X$ such that

(*) $Y^{*} \cup Z \cup\{p\}$ is inconsistent,

(**) $\left.Y^{*} \cup Z \cup\{\neg p\}, Y^{*} \cup Z\right\urcorner \cup\{p\}$ and $\left.Y^{*} \cup Z\right\urcorner \cup\{\neg p\}$ are consistent.

Consider the profile $\left(A_{1}, \ldots, A_{n}\right)$ given (in our notation) by

$$
A_{i}= \begin{cases}\overline{Y^{*} \cup Z \cup\{\neg p\}} & \text { if } i \in C \\ \overline{\left.Y^{*} \cup Z\right\urcorner} & \text { if } i \in \widetilde{C} \backslash C^{\prime} \\ \overline{\left.Y^{*} \cup Z\right\urcorner \cup\{p\}} & \text { if } i \in C^{\prime} \\ \overline{Y^{*}} & \text { if } i \notin C \cup \widetilde{C} .\end{cases}
$$

This profile is in $\left(\mathcal{C}^{*}\right)^{n}$, by Lemma 10 and (**). We have $Y^{*} \subseteq F\left(A_{1}, \ldots, A_{n}\right)$ because $N$ is winning against $\emptyset$ by unanimity-preservation and weak systematicity. Further, for all $z \in Z$, as by $\left({ }^{*}\right) Y^{*}$ is consistent with $z$ and with $\neg z, \overline{Y^{*}}$ contains neither $z$ nor $\neg z$; and so $N_{z}=C$ and $N_{\neg z}=\widetilde{C}$, whence $Z \subseteq F\left(A_{1}, \ldots, A_{n}\right)$ as $C$ is winning against $\widetilde{C}$. By $Y^{*} \cup Z \subseteq F\left(A_{1}, \ldots, A_{n}\right)$ and $\left.{ }^{*}\right), F\left(A_{1}, \ldots, A_{n}\right) \vDash \neg p$, whence by deductive closure $\neg p \in F\left(A_{1}, \ldots, A_{n}\right)$. As by $\left({ }^{* *}\right) Y^{*}$ and $Y^{*} \cup Z^{\urcorner}$are each consistent with $p$ and with $\neg p$, none of $\overline{Y^{*}}$ and $\overline{Y^{*} \cup Z^{\urcorner}}$contains $p$ or $\neg p$; and so $N_{p}=C^{\prime}$ and $N_{\neg p}=C$. So, using weak systematicity, $C$ is semi-winning against $C^{\prime}$, as desired. 
(b) Let $X$ satisfy (i) and (ii), and consider any $C^{\prime} \subseteq N$. We show that $C$ is semi-winning against $C^{\prime}$. This is vacuously true if $C \cap C^{\prime} \neq \emptyset$ (using universality*). Now suppose $C \cap C^{\prime}=\emptyset$. As $C^{\prime} \subseteq N \backslash C$, it suffices by part (a) to show that $C$ is winning against $N \backslash C$.

By (i) there are pairwise disjoint sets $Y^{*}, Z_{1}, Z_{2},\{p\} \subseteq X$ such that

(*) $Y^{*} \cup Z_{1} \cup Z_{2} \cup\{p\}$ is inconsistent;

(**) $Y^{*} \cup Z_{1} \cup Z_{2} \cup\{p\}, Y^{*} \cup Z_{1} \cup Z_{2} \cup\{p\}$ and $Y^{*} \cup Z_{1} \cup Z_{2} \cup\{\neg p\}$ are consistent.

Let $\left(A_{1}, \ldots, A_{n}\right)$ be the profile given by

$$
A_{i}= \begin{cases}\overline{Y^{*} \cup Z_{1} \cup Z_{2} \cup\{\neg p\}} & \text { if } i \in C \\ \overline{Y^{*} \cup\{p\}} & \text { if } i \in N \backslash C .\end{cases}
$$

As in part (a), this profile belongs to $\left(\mathcal{C}^{*}\right)^{n}$ (using Lemma 10 and $\left(^{* *}\right)$ ), and $Y^{*} \subseteq F\left(A_{1}, \ldots, A_{n}\right)$ (as $N$ is winning against $\emptyset$ by unanimity-preservation and weak systematicity). Further, for all $z \in Z_{1} \cup Z_{2}, Y^{*} \cup\{p\}$ is by (**) consistent with $z$ and with $\neg z$, whence $\overline{Y^{*} \cup\{p\}}$ contains neither $z$ nor $\neg z$, and so $N_{z}=C$ and $N_{\neg z}=\emptyset$. So, as $C$ is by part (a) winning against $\emptyset, Z_{1} \cup Z_{2} \subseteq F\left(A_{1}, \ldots, A_{n}\right)$. By $Y^{*} \cup Z_{1} \cup Z_{2} \subseteq F\left(A_{1}, \ldots, A_{n}\right)$ and $(*), F\left(A_{1}, \ldots, A_{n}\right) \vDash \neg p$, so that by deductive closure $\neg p \in F\left(A_{1}, \ldots, A_{n}\right)$. So, by $N_{\neg p}=C$ and $N_{p}=N \backslash C$ and by weak systematicity $C$ is winning against $N \backslash C$, as desired.

Proof of Theorem $1^{*}$. Let $X$ be as specified. Oligarchies satisfy all properties mentioned (using Lemma 4). Now let $F$ have these properties. As $F$ is weakly systematic, $F$ is given, for all $\left(A_{1}, \ldots, A_{n}\right) \in\left(\mathcal{C}^{*}\right)^{n}$, by

$$
F\left(A_{1}, \ldots, A_{n}\right)=\left\{p \in X: N_{p} \text { is semi-winning against } N_{\neg p}\right\} .
$$

So $F$ is oligarchic* if there is a non-empty set $M \subseteq N$ such that

$$
C \text { is semi-winning against } C^{\prime} \Leftrightarrow M \subseteq C \text {, for all disjoint } C, C^{\prime} \subseteq N \text {. }
$$

To show this, note first that the aggregation function $\left.F\right|_{\mathcal{C}^{n}}$, obtained by restricting $F$ to the domain $\mathcal{C}^{n}$, is by part (a) of Theorem 2 oligarchic, say with set of oligarchs $M$. We show that this set $M$ satisfies (6). For any disjoint $C, C^{\prime} \subseteq N$, $C$ is semi-winning against $C^{\prime}$ if and only if $C$ is semi-winning against $N \backslash C$, by Lemma 11 (and using that (i) holds by Lemma 8). The latter is equivalent to $C$ being semi-winning under $\left.F\right|_{\mathcal{C}^{n}}$ (using that $N_{\neg p}=N \backslash N_{p}$ for all $\left(A_{1}, \ldots, A_{n}\right) \in \mathcal{C}^{n}$ and all $p \in X)$, which is in turn equivalent to $M \subseteq C$ as $\left.F\right|_{\mathcal{C}^{n}}$ is the $M$-oligarchy.

Theorem $2^{*}$ follows from Theorem $1^{*}$ with the help of Lemma 8 (which ensures that $X$ satisfies (i)) and the following lemma (which ensures that $F$ is weakly systematic). 
Lemma 12 Let $F$ be universal*, deductively closed, unanimity-preserving and weakly independent.

(a) For all $p, q \in X$, if $C \subseteq N$ is semi-winning against $C^{\prime} \subseteq N$ for $p$, and $p \vDash^{*} q$, then $C$ is semi-winning against $C^{\prime}$ for $q$.

(b) If $X$ satisfies (iii), $F$ is weakly systematic.

Proof (with similarities to the proof of Lemma 9). Let $F$ be as specified.

(a) Consider $p, q \in X$. Suppose $C \subseteq N$ is semi-winning for $p$ against $C^{\prime} \subseteq N$ and $p \vDash^{*} q$. If $C \cap C^{\prime} \neq \emptyset$, it is vacuously true that $C$ is semi-winning against $C^{\prime}$ for $q$. Now let $C \cap C^{\prime}=\emptyset$. By $p \vDash^{*} q$, there is a $Y \subseteq X$ such that $\{p\} \cup Y \vDash q$, and $\{p\} \cup Y$ and $\{\neg q\} \cup Y$ are consistent. So, as $\{p, \neg q\} \cup Y$ is inconsistent,

$\left(^{*}\right)\{p, q\} \cup Y$ and $\{\neg p, \neg q\} \cup Y$ are consistent.

Let $\left(A_{1}, \ldots, A_{n}\right)$ be the profile given (in the above notation) by

$$
A_{i}= \begin{cases}\overline{\{p, q\} \cup Y} & \text { if } i \in C \\ \overline{\{\neg p, \neg q\} \cup Y} & \text { if } i \in C^{\prime} \\ \bar{Y} & \text { if } i \notin C \cup C^{\prime} .\end{cases}
$$

This profile is in $\left(\mathcal{C}^{*}\right)^{n}$, by $\left(^{*}\right)$ and Lemma 10. Further, $\bar{Y}$ contains none of $p, \neg p, q, \neg q$ : otherwise $Y$ would be inconsistent with (another) one of them, violating (*). It follows that $N_{p}=N_{q}=C$ and $N_{\neg p}=N_{\neg q}=C^{\prime}$. So, as $C$ is semi-winning against $C^{\prime}$ for $p, p \in F\left(A_{1}, \ldots, A_{n}\right)$. By unanimity-preservation and weak independence, $Y \subseteq F\left(A_{1}, \ldots, A_{n}\right)$. So $\{p\} \cup Y \subseteq F\left(A_{1}, \ldots, A_{n}\right)$. Hence, by $\{p\} \cup Y \vDash q$ and deductive closure, $q \in F\left(A_{1}, \ldots, A_{n}\right)$. So, as $N_{q}=C$ and $N_{\neg q}=C^{\prime}$, and by weak independence, $C$ is semi-winning against $C^{\prime}$ for $q$, as desired.

(b) Let $X$ satisfy (iii). To show weak systematicity, consider any $p, q \in X$ and $\left(A_{1}, \ldots, A_{n}\right),\left(A_{1}^{*}, \ldots, A_{n}^{*}\right) \in\left(\mathcal{C}^{*}\right)^{n}$ such that $C:=\left\{i: p \in A_{i}\right\}=\left\{i: q \in A_{i}^{*}\right\}$ and $C^{\prime}:=\left\{i: \neg p \in A_{i}\right\}=\left\{i: \neg q \in A_{i}^{*}\right\}$. We suppose that $p \in F\left(A_{1}, \ldots, A_{n}\right)$ and prove that $q \in F\left(A_{1}^{*}, \ldots, A_{n}^{*}\right)$ (the converse being analogous).

First suppose that $p$ or $q$ is non-contingent, i.e. a tautology or contradiction. Then, as all $A_{i}$ and $A_{i}^{*}$ are consistent and deductively closed, one of $C, C^{\prime}$ is $N$ and the other one is $\emptyset$. It is not possible that $C=\emptyset$ and $C^{\prime}=N$ : otherwise $p \notin F\left(A_{1}, \ldots, A_{n}\right)$, since $\emptyset$ is not semi-winning against $N$ for $p$ by unanimity-preservation and weak independence. So $C=N$ and $C^{\prime}=\emptyset$. Then, as desired, $q \in F\left(A_{1}^{*}, \ldots, A_{n}^{*}\right)$, because $N$ is semi-winning against $\emptyset$ for $q$, again by unanimity-preservation and weak independence.

Now let $p$ and $q$ be contingent. Then, by (iii), there are $p=p_{1}, p_{2}, \ldots, p_{k}=$ $q \in X$ such that $p_{1} \vDash^{*} p_{2}, p_{2} \vDash^{*} p_{3}, \ldots, p_{k-1} \vDash^{*} p_{k}$. By $p \in F\left(A_{1}, \ldots, A_{n}\right)$ and weak independence, $C$ is semi-winning against $C^{\prime}$ for $p=p_{1}$. So a simple induction using part (a) tells us that $C$ is semi-winning against $C^{\prime}$ for $p_{k}=q$. Hence $q \in F\left(A_{1}^{*}, \ldots, A_{n}^{*}\right)$, as desired.

We now give constructive proofs of each part of Theorem 3 . 
Proof of Theorem 3. Let $n \geq 3$ and let $X$ contain a contingent proposition.

(a) Now let $F:=F_{\mathcal{W}}$ where $\mathcal{W}$ is defined as $\mathcal{W}=\{C \subseteq N:\{1,3\} \subseteq C$ or $\{2,3\} \subseteq C\}$. Then, by Lemma $5, F$ is non-oligarchic, universal, systematic, unanimity-preserving, and monotonic. We assume that $F$ is not deductively closed, i.e. there is a profile $\left(A_{1}, \ldots, A_{n}\right) \in \mathcal{C}^{n}$ and a $q \in X \backslash F\left(A_{1}, \ldots, A_{n}\right)$, such that $F\left(A_{1}, \ldots, A_{n}\right) \vDash q$. We prove that (i) holds for

$$
\begin{aligned}
& Y:=F\left(A_{1}, \ldots, A_{n}\right) \cup\{\neg q\}, p:=\neg q, \\
& Z_{i}:=\left\{r \in X: N_{r} \cap\{1,2,3\}=\{i, 3\}\right\} \text { for } i=1,2 .
\end{aligned}
$$

First, $Y$ is inconsistent, as $F\left(A_{1}, \ldots, A_{n}\right) \vDash q$.

Second, we show the pairwise disjointness of the sets $Z_{1}, Z_{2},\{p\}$. Obviously, $Z_{1} \cap Z_{2}=\emptyset$. As $F\left(A_{1}, \ldots, A_{n}\right) \subseteq A_{3}$, we have (9). Now $\{p\}$ is disjoint with each $Z_{i}$, because otherwise $p \in Z_{i}$, hence $p \in F\left(A_{1}, \ldots, A_{n}\right)$, so that $F\left(A_{1}, \ldots, A_{n}\right)$ would entail $p$ and also entail $q=\neg p$, in contradiction to (9).

Finally, we have to show the consistency of each of $Y_{\neg Z_{1}}, Y_{\neg Z_{2}}$ and $Y_{\neg\{p\}}$. As $Y=F\left(A_{1}, \ldots, A_{n}\right) \cup\{p\}$ is a disjoint union (by an argument like the previous one),

$$
Y_{\neg\{p\}}=F\left(A_{1}, \ldots, A_{n}\right) \cup\{\neg p\}=F\left(A_{1}, \ldots, A_{n}\right) \cup\{q\} .
$$

By $F\left(A_{1}, \ldots, A_{n}\right) \subseteq A_{3}$ and $F\left(A_{1}, \ldots, A_{n}\right) \vDash q$, we have $F\left(A_{1}, \ldots, A_{n}\right) \cup\{q\} \subseteq A_{3}$, i.e. $Y_{\neg\{p\}} \subseteq A_{3}$. Hence $Y_{\neg\{p\}}$ is consistent. Further, as $3 \in N_{q}$ and (by $q \notin$ $\left.F\left(A_{1}, \ldots, A_{n}\right)\right) N_{q} \notin \mathcal{W}$, we have $1,2 \notin N_{q}$, whence

$$
1,2 \in N_{\neg q}=N_{p} .
$$

Letting $Z_{3}:=\left\{r \in X: N_{r} \cap\{1,2,3\}=\{1,2,3\}\right\}$, we have $Y=Z_{1} \cup Z_{2} \cup Z_{3} \cup\{p\}$, where this is a disjoint union (by an argument like the one above). So

$$
Y_{\neg Z_{1}}=\left\{\neg r: r \in Z_{1}\right\} \cup Z_{2} \cup Z_{3} \cup\{p\} .
$$

Here, $r \in Z_{1}$ implies $r \notin A_{2}$, which implies $\neg r \in A_{2}$. Using this and (7), the relation (8) implies that $Y_{\neg Z_{1}} \subseteq A_{2}$, whence $Y_{\neg Z_{2}}$ is consistent. For analogous reasons, $Y_{\neg Z_{2}}$ is consistent.

(b) Let $F$ be $F_{\mathcal{W}}$ where $\mathcal{W}:=\{N, N \backslash\{1,2\}\}$. By Lemma $5, F$ is nonmonotonic (hence non-oligarchic), universal, systematic, and unanimity-preserving (the latter uses that $\emptyset \notin \mathcal{W}$ by $n \geq 3$ ). The crucial claim is that, if (ii) is violated, $F$ is deductive closed. We suppose $F$ is not deductively closed and prove (ii).

By assumption, there is a profile $\left(A_{1}, \ldots, A_{n}\right) \in \mathcal{C}^{n}$ and a $q \in X \backslash F\left(A_{1}, \ldots, A_{n}\right)$, such that $F\left(A_{1}, \ldots, A_{n}\right) \vDash q$. We prove that (ii) hods for

$$
\begin{aligned}
& Y:=\left\{r \in X: N_{r}=N \backslash\{1,2\} \text { or } N_{r}=N\right\} \cup\{\neg q\}\left(=F\left(A_{1}, \ldots, A_{n}\right) \cup\{\neg q\}\right) \\
& Z:=\left\{r \in X: N_{r}=N \backslash\{1,2\}\right\}, p:=\neg q .
\end{aligned}
$$

First, $Y$ is inconsistent as $F\left(A_{1}, \ldots, A_{n}\right) \vDash q$. 
Second, we show that $\{p\}(=\{\neg q\})$ and $Z$ are disjoint. Note that

$$
F\left(A_{1}, \ldots, A_{n}\right) \text { is consistent, }
$$

as $F\left(A_{1}, \ldots, A_{n}\right) \subseteq \cap_{k \in N \backslash\{1,2\}} A_{k}$. If $\{p\}$ and $Z$ were not disjoint, we would have $p \in Z$, hence $p \in F\left(A_{1}, \ldots, A_{n}\right)$; so $F\left(A_{1}, \ldots, A_{n}\right)$ would entail both $p(=\neg q)$ and $q$, violating (9).

Finally, we show that $Y_{\neg Z}$ and $Y_{\neg(Z \cup\{\neg q\})}$ are consistent. Note that $\cap_{k \in N \backslash\{1,2\}} A_{k} \vDash q$ by $F\left(A_{1}, \ldots, A_{n}\right) \vDash q$ and $F\left(A_{1}, \ldots, A_{n}\right) \subseteq \cap_{k \in N \backslash\{1,2\}} A_{k}$. Hence for each $k \in N \backslash\{1,2\}, A_{k}$ entails $q$, hence contains $q$. So $N \backslash\{1,2\} \subseteq N_{q}$. Hence, as $N_{q}$ is (by $q \notin F\left(A_{1}, \ldots, A_{n}\right)$ ) neither $N$ nor $N \backslash\{1,2\}, N_{q}$ is either $N \backslash\{1\}$ or $N \backslash\{2\}$. We assume that

$$
N_{q}=N \backslash\{1\} \text {, and hence } N_{\neg q}=\{1\}
$$

(the case of $N_{q}=N \backslash\{2\}$ being analogous). Note that

$$
\begin{aligned}
Z \cup\{p\} & =\{\neg q\} \cup\left\{r \in X: N_{r}=N \backslash\{1,2\}\right\} \\
Y & =\{\neg q\} \cup\left\{r \in X: N_{r}=N \backslash\{1,2\}\right\} \cup\left\{r \in X: N_{r}=N\right\}
\end{aligned}
$$

where these are unions of pairwise disjoint sets by $N_{\neg q}=\{1\}$. So

$$
\begin{aligned}
Y_{\neg Z}= & \left.\left(\{\neg q\} \cup\left\{r \in X: N_{r}=N \backslash\{1,2\}\right\} \cup\left\{r \in X: N_{r}=N\right\}\right\}\right)_{\neg\left\{r \in X: N_{r}=N \backslash\{1,2\}\right\}} \\
= & \{\neg q\} \cup\left\{\neg r \in X: N_{r}=N \backslash\{1,2\}\right\} \cup\left\{r \in X: N_{r}=N\right\}, \\
Y_{\neg(Z \cup\{p\})}= & \left(\{\neg q\} \cup\left\{r \in X: N_{r}=N \backslash\{1,2\}\right\} \cup\left\{r \in X: N_{r}=N\right\}\right)_{\neg\left(\{\neg q\} \cup\left\{r \in X: N_{r}=N \backslash\{1,2\}\right\}\right)} \\
= & \{q\} \cup\left\{\neg r \in X: N_{r}=N \backslash\{1,2\}\right\} \cup\left\{r \in X: N_{r}=N\right\} .
\end{aligned}
$$

It follows that $Y_{\neg Z} \subseteq A_{1}$ and $Y_{\neg(Z \cup\{p\})} \subseteq A_{2}$, in both cases using (10) and $N_{r}=N \backslash\{1,2\} \Leftrightarrow N_{\neg r}=\{1,2\}$. So $Y_{\neg Z}$ and $Y_{\neg(Z \cup\{p\})}$ are consistent.

(c) Suppose $X$ violates (iii). Then there is a contingent $r \in X$ with no $\vDash^{*}$-path to some $s \in X$. Write $X=X_{1} \cup X_{2}$, where

$$
X_{1}:=\left\{s \in X \text { : there is a } \vDash^{*} \text {-path from } r \text { to } s\right\} \text { and } X_{2}:=X \backslash X_{1} .
$$

Let $F$ be the universal aggregation function given, for all $\left(A_{1}, \ldots, A_{n}\right) \in \mathcal{C}^{n}$, by

$$
F\left(A_{1}, \ldots, A_{n}\right):=\left(X_{1} \cap A_{1}\right) \cup\left[X_{2} \cap\left(\cap_{i \in N} A_{i}\right)\right] ;
$$

i.e. within $X_{1}$ person 1 is a dictator and within $X_{2}$ the unanimity function is used. $F$ is non-oligarchic (by $X_{1} \neq \emptyset$ and $X_{2} \neq \emptyset$ ), universal, unanimitypreserving, and independent.

To see monotonicity, let $\left(A_{1}, \ldots, A_{n}\right),\left(A_{1}^{*}, \ldots, A_{n}^{*}\right) \in \mathcal{C}^{n}$ be such that $A_{i}^{*}=A_{i}$ for all individuals $i$ except from, say, individual $j$, who has $A_{j}^{*}=F\left(A_{1}, \ldots, A_{n}\right)$. To show that $F\left(A_{1}^{*}, \ldots, A_{n}^{*}\right)$ and $F\left(A_{1}, \ldots, A_{n}\right)$ are identical, we show that they 
have the same intersections with $X_{1}$ and with $X_{1}$. Regarding the intersection with $X_{2}$, we have

$$
\begin{aligned}
X_{2} \cap F\left(A_{1}^{*}, \ldots, A_{n}^{*}\right) & =X_{2} \cap\left(\cap_{i \in N} A_{i}^{*}\right) \\
& =X_{2} \cap F\left(A_{1}, \ldots, A_{n}\right) \cap\left(\cap_{i \in N \backslash\{j\}} A_{i}\right) \\
& =X_{2} \cap F\left(A_{1}, \ldots, A_{n}\right),
\end{aligned}
$$

as desired. Regarding the intersection with $X_{1}$, we have again

$$
X_{1} \cap F\left(A_{1}^{*}, \ldots, A_{n}^{*}\right)=X_{1} \cap A_{1}^{*}=X_{1} \cap F\left(A_{1}, \ldots, A_{n}\right),
$$

where the last equality follows from $A_{1}^{*}=F\left(A_{1}, \ldots, A_{n}\right)$ if $j=1$, and from $X_{1} \cap A_{1}^{*}=X_{1} \cap A_{1}=X_{1} \cap F\left(A_{1}, \ldots, A_{n}\right)$ if $j \neq 1$.

We finally show deductive closure. We suppose for a contradiction that there is a profile $\left(A_{1}, \ldots, A_{n}\right) \in \mathcal{C}^{n}$ and a $q \in X \backslash F\left(A_{1}, \ldots, A_{n}\right)$, such that $F\left(A_{1}, \ldots, A_{n}\right) \vDash q$. By $F\left(A_{1}, \ldots, A_{n}\right) \subseteq A_{1}$, we have (9), and we have $A_{1} \vDash q$, hence $q \in A_{1}$. So $q \in X_{2}$ : otherwise $q$ would be in $X_{1} \cap A_{1}$, hence in $F\left(A_{1}, \ldots, A_{n}\right)$, hence entailed by $F\left(A_{1}, \ldots, A_{n}\right)$. As $X$ is finite or the logic compact, $F\left(A_{1}, \ldots, A_{n}\right)$ has a minimal subset $Z$ that entails $q$. There is a $p \in Z \cap X_{1}$ : otherwise $Z \subseteq X_{2}$, hence $Z \subseteq \cap_{i \in N} A_{i}$, so that $\cap_{i \in N} A_{i} \vDash q$, whence (by Lemma 4) $q \in \cap_{i \in N} A_{i} \subseteq F\left(A_{1}, \ldots, A_{n}\right)$, a contradiction.

We show that $p \vDash^{*} q$, a contradiction by $p \in X_{1}$ and $q \in X_{2}$. Putting $Y:=Z \backslash\{p\}$, we have $\{p\} \cup Y=Z \vDash q$, where $Y$ is consistent with $\neg q$ (otherwise $Y \vDash q)$ and with $p$ (as $Z$ is consistent by $Z \subseteq F\left(A_{1}, \ldots, A_{n}\right)$ ). 